\title{
Numerical Simulation of Blank-making Roll Forging Process for Heavy Automotive Front Axle
}

\author{
XIA Hua \\ Department of Materials Forming and \\ Control Engineering, Chongqing \\ University of Technology \\ Chongqing, China
}

\author{
GUO Xiao-long \\ Department of Materials Forming and \\ Control Engineering, Chongqing \\ University of Technology \\ Chongqing, China
}

\author{
JI Cheng-cheng \\ Department of Materials Forming and \\ Control Engineering, Chongqing \\ University of Technology \\ Chongqing, China
}

\begin{abstract}
The numerical simulation of the heavy automobile front axles precision roll forging-die forging forming process was carried out by the DEFORM-3D. The temperature field, stress field, strain field, velocity field and metal flow condition in the forming process have been analyzed. The simulation results provide a theoretical guidance for the actual production of the heavy automobile front axle precision roll forging-die forging forming process, die design and equipment selection.
\end{abstract}

Keywords-front axle, precision roll forging, thermal-mechanical coupling.

\section{INTRODUCTION}

Nowadays, products for various fields in practice have to meet higher quality demands and especially need to be developed in shorter times. However, here is no exact roll forging theory guidance for the heavy automobile front axles precision roll forging-die forging forming process, and we always need to repeatedly modify the process parameters and mold structure. The three dimensional thermo-mechanical coupling simulation of the heavy automobile front axles precision roll forging-die forging forming process is carried out by the DEFORM-3D to analyze and solve the problems encountered in the forming process. According to the problems occurred in the simulation,this paper made corresponding measures to optimize the process parameters and the mold structure of heavy automobile front axle precision roll forging. Analyse the situation of metal flow, the temperature field and strain field in whole forming process to provide the theory basis and guidance to actual production of the heavy automobile front axle precision roll forging-die forging forming process, die design and equipment selection $^{[1-4]}$.

During the three-dimensional finite element simulation of forging process, the temperature fields will be affected by several factors.It includes the mechanical performance of material, thermodynamic properties, the speed of deformation during the forming process and the coefficient of friction ${ }^{[5]}$. So we are using the three-dimensional finite element thermal coupling simulation for heavy automobile front axles precision roll forging - forging forming process.

Roll forging is a process for reducing the cross-sectional area of heated bars or billets by passing them between two driven rolls that rotate in opposite directions and have one or more matching groovesin each roll ${ }^{[6]}$,as shown in Figure 1. This process reduces the cross-sectional area of the bar while changing its shape. It is a very cost effective and property effective process that can be applied as a final forming operation or a preliminary forming operation, followed by other forging processes in components manufacture, such as hand shovels, spades, various agricultural tools, crankshafts connecting rods, and other automotive parts.

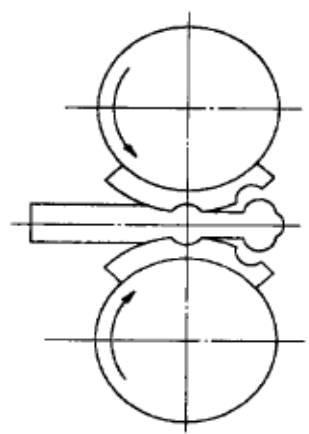

Figure.1 The schematic for roll forging process

\section{FINITE ELEMENT SIMULATION RESULTS AND ANALYSIS}

\section{A. Conditions of the Simulation}

A commercial 42CrMo steel was used in this investigation. Its initial cross-section diameter is $150 \mathrm{~mm}$, the length is $965 \mathrm{~mm}$, and initial temperature is $1150{ }^{\circ} \mathrm{C}$. Using a rigid-plastic material law, the isothermal 3-D simulation of the roll forging process was performed using the commercial software Deform-3D. The flow stress of the material is the function of the accumulated strain, the instantaneous strain rate, and the current temperature, which can be read directly from the materials in the software. For the calculation of the friction stress, the law of constant friction (the friction factor model), which assumes a proportionality between the friction stress and the shear yield stress, was used.

\section{B. The blank deformation analysis}

According to the simulation results in Figure.2, large changes are occurred in the transverse dimension of the blank in the entire molding process, but a small change in the longitudinal dimension. After forging, bending and whole 
finish forging, blank flow direction and the distribution is shown in Figure 2.

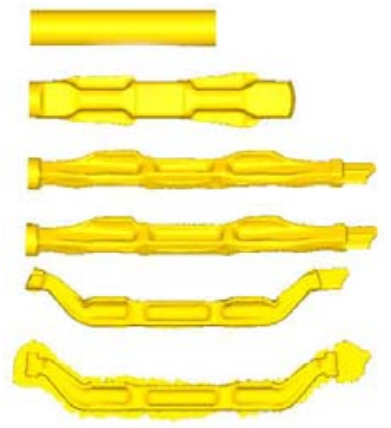

Figure 2 The blank change in the whole forming process

There are some different problems in the whole forming process and different measures were taken to solve the corresponding problems. For example, In the second pre-forming forging,by adding guide groove solves the swinging which is produced by the inhomogeneous deformation of the blank in the simulation process. By adding the longitudinal length of the cross section in the transition section solves the serious distortion of the second preform roller forgings parts of the spring abutment. By determining slip the value of each feature section's and constantly modifying the length of the roll forging dies to make the blank and die-slot get a reasonable engagement and control the longitudinal length of the forgings. The ultimate goal is to get qualified the heavy automobile front axles.

\section{The blank temperature analysis}

In the actual production process, it is better to ensure the metallic material to produce plastic deformation at higher temperature. Each forming step interval should be reduced in these multi-step metal material plastic forming. The change in temperature of the blank is affected by forging forming, bending forming and whole finish forging in the finite element thermal coupling simulation. Blank changes of the temperature field in the entire precision roll forging - forging forming process, as shown in Figure 3.

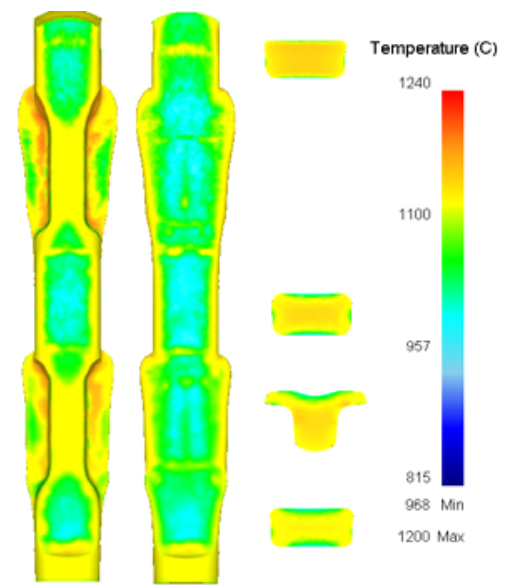

(a).The temperature fields for the first roll forging

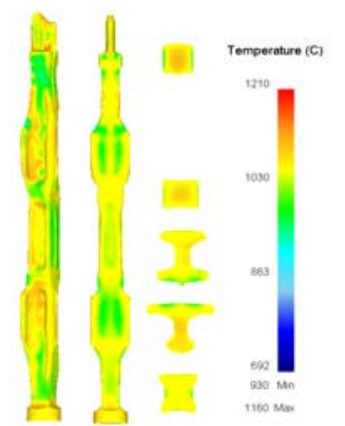

(b).The temperature fields for the second roll forging

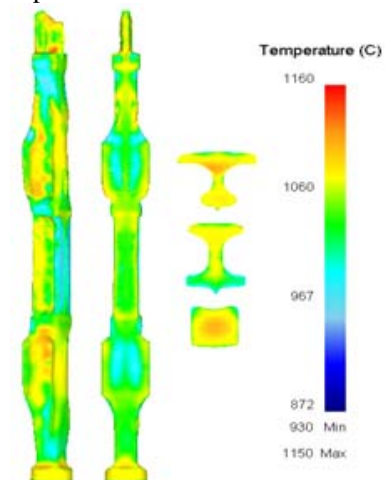

(c).The temperature fields for the third roll forging

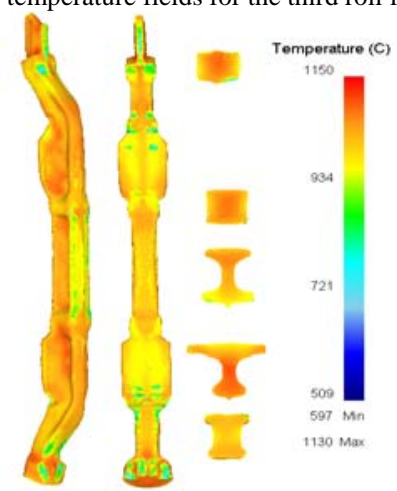

(d) The temperature fields for bend forming

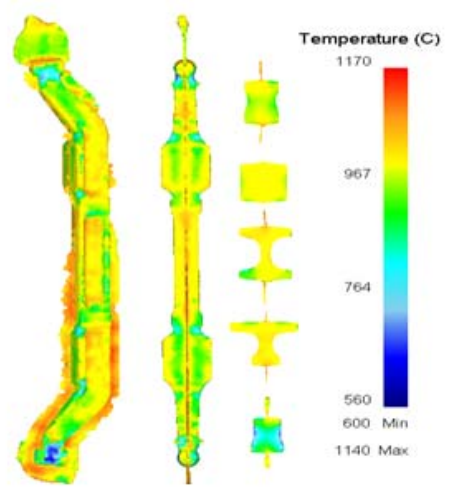

(e).The temperature fields for final forging

Figure 3 The blanks temperature distribution in whole forming process

The blank temperature field in the vertical and horizontal distribution is uneven from the changes in temperature field situation in the front axle precision roll forging - die forging process. It is due to the result affected by the plastic 
deformation and frictional.

There is thermal conductivity between blank and mold, air convection as well as thermal radiation makes blanks' temperature decreased. Blank surface directly contact with the mold and the air, so they have different temperature. The heat transfers is faster from blank to air thus causing the blank surface temperature decreased rapidly. Also there is temperature gradient between heart and the surface, the circulation makes the blanks' temperature getting lower and lower.

As we can see from the changes in temperature field ,The temperature of the blank decreases from the initial $1150^{\circ} \mathrm{C}$ to the final average $950{ }^{\circ} \mathrm{C}$. It is reduced by about $200{ }^{\circ} \mathrm{C}$ compared to the initial temperature. For 42CrMo steel,the forging temperature should be more than $850{ }^{\circ} \mathrm{C}$,so the blank can be completely formed in the heavy automobile front axle, precision roll forging - forging forming process by once heated.

\section{The blank equivalent strain analysis}

The change in equivalent strain of the blank is affected by forging forming, bending forming and whole finish forging in the finite element thermal coupling simulation. The change of the equivalent strain field for the blank in the entire precision roll forging - forging forming process is shown in Figure 4.

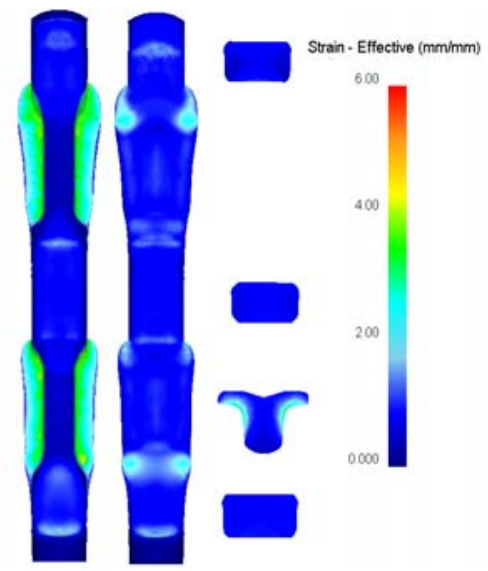

(a).The strain fields for the first roll forging

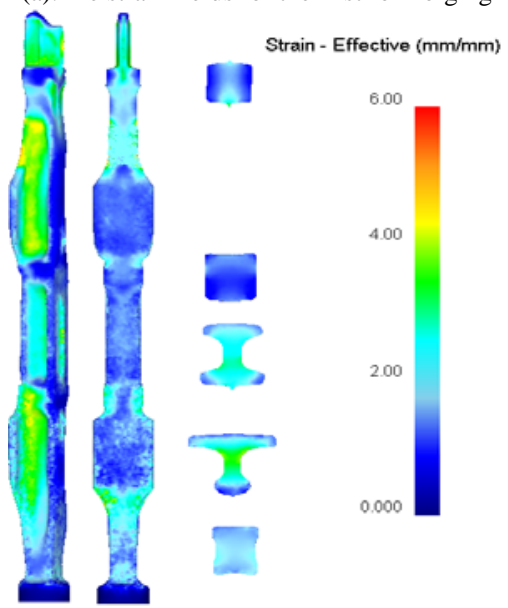

(b).The strain fields for the first roll forging

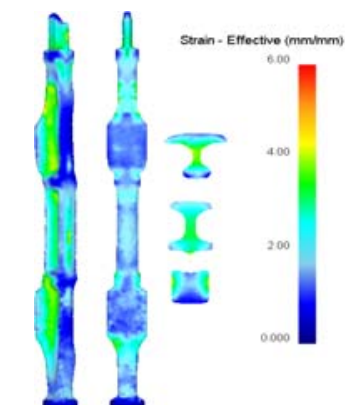

(c).The strain fields for the third roll forging

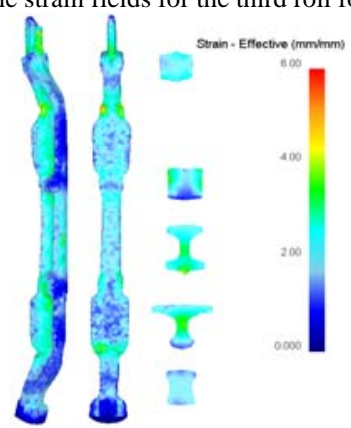

(d) The strain fields for bend forming

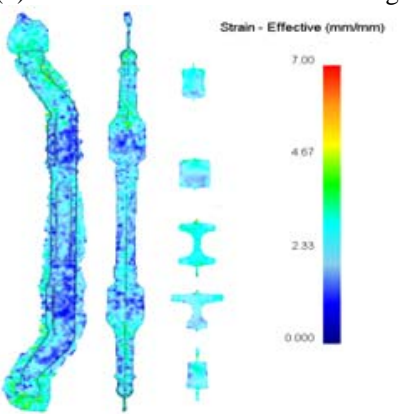

(e).The strain fields for final forging

Figure 4 Equivalent strain field in the whole forming process

As can be seen from Figure 4 (a), when blank is fogged in the first roll forging preform, because the mandatory is wide spreaded by the mold structure of the hat grooved portion of the spring abutment, the equivalent strain of the spring abutment portion is the largest. Intermediate-beams, cantilevered I-beam, box, fist parts by circular blank roll forging formed into symmetrical shape of the box-type structure, these parts bear smaller equivalent strain.

In Figure 4 (b), we can see that, when the blank is fogged in the second pre-forming roll forging, since the spring abutment, the intermediate joist, and parts of the intermediate portion of the cantilever joist dough should be changed by the symmetrical box-type structure for the joist shape, the middle of the blank is subjected to compression around the blank squeezed by the mold, so these parts of the larger equivalent strain; box, and the structure of the fist portion is unchanged, so these parts has a smaller equivalent strain.

As can be seen from Figure 4 (c), the blank in the third end roll forging, spring abutment, cantilevered I-beam, box, fist parts almost no deformation. This process forming part of the intermediate joist.The intermediate joist parts of the blank 
and roll forging die contact area is large, so the deformation space is relatively small, the blank deformed larger and more uniform by role of upsetting extrusion.

From Figure 4 (d), it can be seen that, during the bend-shaping, the deformation of the blank mainly concentrated on the bending deformation region.Large plastic deformation occurred in this region, so the equivalent strain of the bending parts is greater.

From Figure 4 (e) it can be seen, when the blank is under the overall final forging, since only the fist portion of both sides was formed by die forging, the deformation is a bit larger, while the rest of the parts is just shaping with a small deformation.Both ends of the fist, and flash-generating parts of in the fists of both sides and some parts with overlap ,the equivalent strain is a bit larger.

\section{CONCLUSION}

Through three-dimensional finite element simulation, the flow state, strain field and temperature field during the blank forming process is analyzed in this paper. According to the simulation results, the process parameters of precision roll forging - forging was optimized and mold structure is improved, which provides a theoretical basis for the research and development of the process.

\section{REFERENCES}

[1] Wang Jin-lu, Wang Gao-cao. Thermal-coupled FEM Analysis of Precision Roll Forging Performing of Front Axle [J]. New Technology \& New Process. Digital design and manufacturing, 2006, 3: 19-21.

[2] Xu Chun-guo Ma Cui-ping and Ren Guang-sheng, et al. Thermal coupling finite-element analysis of axial feed bar rolling [J]. Journal of Plasticity Engineering, 2004, 12(6): 80-83.

[3] Zeng Zhi-peng, Liu Xiao-fei and Jin Quanlin, et al. Simulation and optimizition of extrusion process for half-shaft of automobile [J]. Forging \& Stamping Technology, 2002, 6: 7-10.

[4] Hong Sukmoo, Lee Seungyoon, Kim Naksoo. A parametric study on forming length in roll forming [J]. Journal of Materials Processing Technology, 2001, 113(1-3): 774-778.

[5] Iankov R. Finite element simulation of profile rolling of wire [J]. Journal of Materials Processing Technology, 2003, 142(2): 355-361.

[6] G.D. La hoti, ASM Ha ndbook (Vol.14, The Ma te ria l Inte rna tiona Soc ie ty, Ohio, 2001) p.1. 